Article

\title{
Gap-Dependent Localized High Energy Multiple Dipolar Modes in Passive Silver-Coated Silica Nanoparticle Antennas
}

\author{
Atta Ur Rahman ${ }^{1}$, Junping Geng ${ }^{1, *}$, Sami Ur Rehman ${ }^{1}$, Khizar Hayat ${ }^{2}$, Xianling Liang ${ }^{1}$ \\ and Ronghong Jin ${ }^{1}$ \\ 1 Department of Electronic Engineering, Shanghai Jiao Tong University, Shanghai 200240, China; \\ attaur@sjtu.edu.cn (A.U.R.); samiurrehman101@yahoo.com (S.U.R.); \\ liangxl@sjtu.edu.cn (X.L.); rhjin@sjtu.edu.cn (R.J.) \\ 2 Department of Physics, Abdul Wali Khan University Mardan, Mardan 23200 KPK, Pakistan; \\ Khizar3@gmail.com \\ * Correspondence: gengjunp@sjtu.edu.cn; Tel.: +86-021-342-04663
}

Received: 24 October 2017; Accepted: 2 November 2017; Published: 17 November 2017

\begin{abstract}
The gap-induced plasmonic response of metallic nanoparticles drastically changes the near and far-field properties of nanoparticle antenna. Similar to a pair of metallic nanostructures, the two nanoparticles, with a dielectric core and silver shell in close proximity, exhibit multiple high energy plasmonic resonances at the short wavelength end of their optical spectrum. In this article, we have overwhelmingly investigated the disparity in the electric field of a core-shell dimer antenna when the gap between nanoparticles within the dimer becomes sub-nanometer in length. We used an electromagnetic planewave to excite the core-shell nanoparticles within the dimer. Frequency domain Finite Element Method (FEM) was employed for the numerical optical analysis of a dimer comprised of two silver-coated silica (SCS) nanoparticles in close proximity, using Computer Simulation Technology (CST) Microwave Studio. A modified Drude model has been used to predict the optical properties of the system with incorporating the size effects. The SCS dimer was numerically analyzed in the visible frequency band, and anomalies in near-field plasmonic coupling were investigated in detail. The inter-surface gap $g$ between nanoparticles within the dimer varied in a range from 0.1 to $402 \mathrm{~nm}$.
\end{abstract}

Keywords: core-shell nanoparticles; silver; coupling; near field; Multiple dipolar modes; gap; Plasmons

\section{Introduction}

Light-induced oscillation of conduction electrons within sub-wavelength regimes in metallic nanoparticles such as Ag and $\mathrm{Au}$ is usually termed as localized surface plasmon resonance (LSPR). Owing to their small size, these nanoparticles are able to concentrate light in a sub-wavelength region, resulting in a remarkable enhancement of the electric field in their vicinity [1,2]. The wave dynamics and electron transport studies in the vicinity of and inside complex nanostructures interconnect different fields, such as metamaterials, plasmonic, quantum transport, and molecular electronics $[3,4]$. Properties exhibited by LSPR, e.g., near/far-field enhancement and nanoantenna performance, are tunable by varying certain parameters, such as the particle diameter and the dielectric core and/or shell, and by modifying the surfaces of the nanoparticles [5-7]. The plasmonic behavior of metal and core-shell nanoparticles has been utilized in many advanced technologies, e.g., imaging, sensing, and biomedical technologies [8]. Most of these applications incorporate the optical response of metallic nanoparticle arrays or clusters of nanoantennas. In order to utilize the optical response of these 
nanoparticles, detailed studies of the optical properties of the dimers, trimers, and arrays of many particles are extremely vital [9]. One important parameter is the inter-particle distance for regulating the plasmonic response of metallic and core-shell nanoparticle antennas, according to the necessities of already mentioned applications. Near field dipolar coupling is very sensitive to the gap between two plasmonic nanoparticles within the dimer antenna. Therefore, in recent years, the hybridization of electric fields between two nanostructures excited by Electromagnetic (EM) waves and separated by subwavelength regions has become of interest to researchers working in this area. A strong electric field of two or more metal nanoparticles in close proximity has demonstrated an enhancement in biochemical sensors, nanoantenna, and imaging properties [10-12]. Various types of nano-structures, e.g., nano-rods, bowties, nanowires, nanoparticles, and nano-cubes, have been investigated in attempts to achieve strong electric fields in their closed surroundings [11,13,14]. Different theoretical approaches have been attempted so as to explain the interacting electric fields of two nanostructures in close proximity. These theoretical approaches can be divided into two categories: (i) non-local/quantum optical response and (ii) classical/semi-classical optical response [15]. In 2003, it was reported that the wavelength of an inelastically scattered EM wave varies exponentially with the inter-particle gap and is independent of the shape and size of plasmonic nanostructures [16,17]. Some recent theoretical and experimental studies about nanoparticle dimers have confirmed that exponential behavior, called the "Ruler equation," breaks down when the gap between nanostructures in terms of size becomes smaller [18] since, at such small separation, there is an abrupt increase in near-field coupling of the dimer [19]. In addition to the breakdown of the Ruler equation due to strong near-field coupling, there is an associated transition of electric field distribution which leads to the breeding of two plasmonic modes as the gap between nanoparticles approaches a nanometer or sub-nanometer level [20]. These plasmonic modes strongly affect the major bonding dipolar mode between nanoparticles within the dimer. In recent years, this escalation in near-field coupling and the generation of different dipolar modes have been investigated in various types of pair contacting surfaces, e.g., spherical or flat surfaces of nanowires and face-face or edge-edge nano-cubes $[13,20]$.

Contrary to previous reports, we suggest in this paper that such breeding plasmonic modes can be explained by a classical modified Drude model, which incorporates size effects in terms of collision frequency. In this article, a detailed investigation of near-field coupling of an SCS dimer is presented. Throughout the article, we use the term dimer antenna for two interacting SCS nanoparticles excited by an EM plane wave. In this article the strength of field coupling and directivity of high energy Multiple Dipolar Modes (HEMDMs) has been explained. We investigated the optical properties of two interacting SCS nanoparticles using a modified Drude model which also includes the size effects of nanostructures on their dielectric constant. This study shows that the near-field plasmonic properties of SCS dimers can be tuned by varying the gap between nanoparticles within a dimer, instead of via more complicated processes such as varying the diameter of the core and the surrounding shell. We perform all calculations using a CST Microwave Studio.

\section{Geometry of Dimer Antenna and Modified Drude Model}

All dimensional parameters of the SCS dimer are depicted in Figure 1. The SCS dimer structure was mainly comprised of two SCS nanoparticles, separated by distance " $d$ " and embedded in free space. Each individual particle had a passive silica core with a radius $R_{1}$ of $37 \mathrm{~nm}$ and an Ag shell with a thickness of $12 \mathrm{~nm}$ around the silica core. The total diameter of each particle was $98 \mathrm{~nm}$. The spatial directions $X, Y$ and $Z$ were specified by a local coordinate system $(u, v, w)$, as shown in Figure 1 . An electromagnetic planewave propagated along the Z-axis excited the SCS dimer antenna, while its electric and magnetic components were perpendicular to the direction of wave propagation. The center of the left-handed particle lay at $X=Y=Z=0$, while the other, similar particle on the right hand was always portable by the distance $g$ along the $X$-axis. 


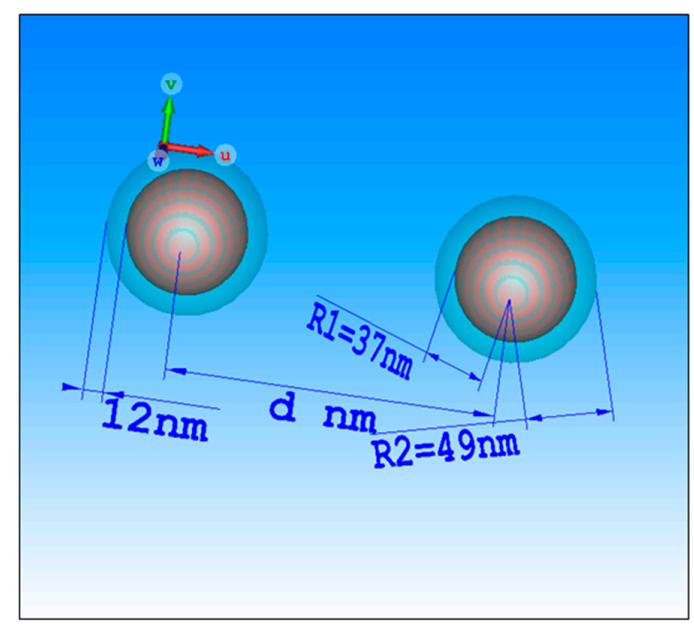

Figure 1. Silver-coated silica (SCS) dimer model. The brown sphere indicates the silica core and the light-blue semi-transparent circle around the silica core represents the silver shell; $d=g+98$ is the separation distance between the centers of the nanoparticles.

Plasmonic resonance and near-field enhancement properties of the core-shell nanoparticle system can be tuned greatly by varying the ratio $R_{1} / R_{2}$ [21]. To investigate the optical response of a nano-sized dimer with a metal shell and a dielectric core, size effects need to be taken into account in the Drude model because the optical response of nano-dimensional materials is intensely different from their bulk counterpart. The Drude model modified with size effects is presented in [21]. The dielectric response of any material can be written in terms of the Drude response and the interband transition, as follows:

$$
\varepsilon(R, \omega)=\varepsilon_{\text {Drude }}(R, \omega)+\chi_{\text {Interband }}(\omega)
$$

where $R$ is the thickness of the surrounding shell. The Drude model can be expressed as

$$
\varepsilon_{\text {Drude }}(R, \omega) 1=\frac{\omega_{p}^{2}}{\Gamma(R)^{2}+\omega^{2}}+j \frac{\Gamma(R)^{2} \omega_{p}^{2}}{\omega\left(\Gamma(R)^{2}+\omega^{2}\right)} .
$$

This modified Drude model incorporates size effects in the form of size-dependent damping frequency. This is due to the fact that, in nano-dimensional materials $(\leq 100 \mathrm{~nm})$ or materials smaller than the mean free path of electrons, the damping frequency ' $\Gamma$ ' has an inverse relation to the size of the material $(R)$ as given in Equation (3).

$$
\Gamma(R)=\Gamma_{\infty}+\frac{A V_{F}}{R} .
$$

$A$ is constant at $\sim 1$, and $V_{F}$ is the Fermi velocity. The values of all Drude parameters and the Fermi velocity were used to compute the dielectric dispersion in a $12 \mathrm{~nm}$ silver shell, where $m^{*} / m=0.96$, $N=5.85 \times 10^{28} \mathrm{~m}^{-3}, V_{F}=1.39 \times 10^{6} \mathrm{~m} / \mathrm{s}$, and $\omega_{p}=1.39269 \times 10^{13} \mathrm{~s}^{-1}$, as given in [21]. It should be noted that this model incorporates size effects but excludes quantum tunneling or charge transfer between SCS nanoparticles at small gaps. The inclusion of size effects transforms the Drude model from a classical to a semi-classical model.

\section{Results and Discussion}

The variation in the scattering cross-section of SCS dimer antennas was monitored as a function of the wavelength at different values of $g(=d-98)$, as depicted in Figure 2a. The gap $g$ varied from 0.1 to $402 \mathrm{~nm}$. Small values of $g(0.1 \sim 2 \mathrm{~nm})$ ensure strong near-field coupling. The number of plasmonic resonance peaks and their intensities were sensitive to inter-particle gap $g$. The SCS dimer 
antennas exhibited a single major dipolar mode when $12 \mathrm{~nm} \leq g \leq 402 \mathrm{~nm}$. A second dipolar mode appeared when the value of $g$ was below $12 \mathrm{~nm}$, and a third dipolar modes was generated when $g \leq 2 \mathrm{~nm}$, as shown in Figure 2b. The latter two dipolar modes we refer to here as high energy Multiple Dipolar Modes (HEMDMs). The corresponding resonance energies and wavelengths of all modes are tabulated in Table 1. In Figure 2a, the first resonance peak is represented by circles and has high intensity and broadness. This resonance peak is attributed to the major bonding dipolar mode and red-shifted as $g$ decreased. Figure $2 b$ shows the presence of HEMDM peaks in a more highlighted form in a $g$ range from $0.1-12 \mathrm{~nm}$. Only this range was selected for Figure $2 \mathrm{~b}$ because HEMDM peaks above this range were not present. The major dipolar mode exhibits greater intensity and broadness than the HEMDM. All three resonance peaks corresponded to different dipolar modes with different resonance wavelengths and red-shifted with a decrease in $g$ value. The most energetic HEMDM peak had resonance energy in a range from 2.434 to $2.805 \mathrm{eV}$ and appeared when $g$ was $0.1-1.1 \mathrm{~nm}$, while the second HEMDM (1.990-2.794 eV) occurred when $g$ was $0.1-12 \mathrm{~nm}$. The peak corresponded to the major dipolar mode and shifted from higher $(2.481 \mathrm{eV})$ to lower energy values $(1.441 \mathrm{eV})$ as the $g$ value decreased from 402 to $0.1 \mathrm{~nm}$. In previous findings, the plasmonic behavior of major dipolar modes was investigated in a core-shell dimer system and was based only on the $R_{1} / R_{2}$ ratio [22]. However, the findings of this article suggest that, by varying the inter-particle distance $g$, the SCS dimer will possess high tunable plasmonic properties regarding the co-existence of different dipolar modes with different frequency and energy values.
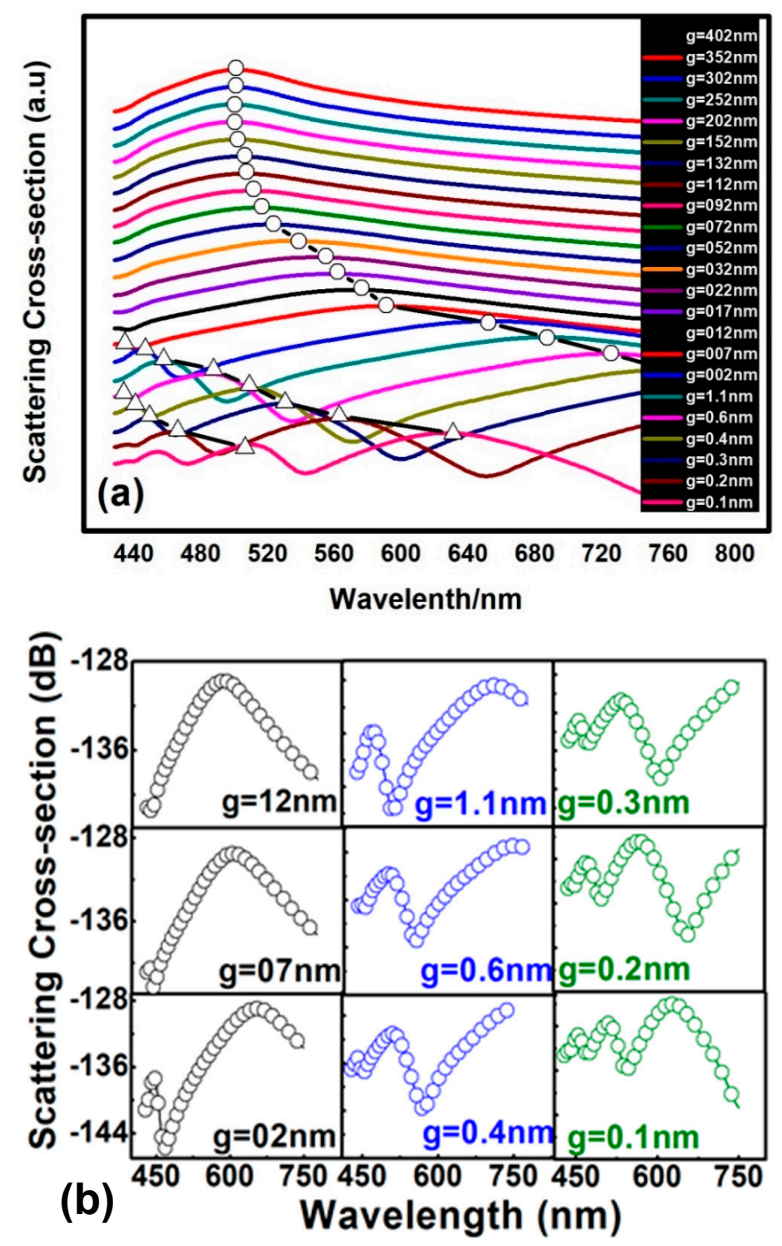

Figure 2. (a) Scattering cross-section of dimer as a function of wavelength at all calculated values of $g$. The circles shows a main dipolar resonance peak, while the triangles indicate high energy multiple modes; (b) High-energy multiple resonance peaks where $g$ is $0.1-12 \mathrm{~nm}$. 
Table 1. Peaks positions at small value of $g$.

\begin{tabular}{ccccccc}
\hline \multirow{2}{*}{ S. No. } & \multirow{2}{*}{$(\mathbf{n m})$} & \multirow{2}{*}{ No. Peaks } & \multicolumn{2}{c}{ Energy (eV) } & \multicolumn{2}{c}{ Wavelength (nm) } \\
\cline { 5 - 7 } 1 & 0.1 & 3 & Main Dipolar Peak & HEMDEM Peaks & MAIN Dipolar Peak & HEMDM Peaks \\
\hline \multirow{2}{*}{2} & 0.4 & 3 & i. 1.441 & $\begin{array}{c}\text { ii. } 1.990 \\
\text { iii. } 2.434\end{array}$ & i. 348.4 & $\begin{array}{c}\text { ii. } 481.1 \\
\text { iii. } 588.5\end{array}$ \\
\hline 3 & 02 & 2 & i. 1.66 & $\begin{array}{l}\text { ii. } 2.406 \\
\text { iii. } 2.805\end{array}$ & i. 401.3 & $\begin{array}{c}\text { ii. } 581.7 \\
\text { iii. } 678.2\end{array}$ \\
\hline 4 & 07 & 2 & i. 2.10 & ii. 2.78 & i. 654.1 & ii. 445.9 \\
\hline 5 & 12 & 2 & i. 2.16 & ii. 2.86 & i. 589.4 & ii. 432.5 \\
\hline 6 & 17 & 1 & i. 2.27 & - & i. 572.5 & i. 430.0 \\
\hline
\end{tabular}

The origin for the presence of three resonance peaks can be better understood by considering the scattering and absorption cross-section as a function of wavelength in a more highlighted form, e.g., when $g=0.4 \mathrm{~nm}$ (Figure 3).

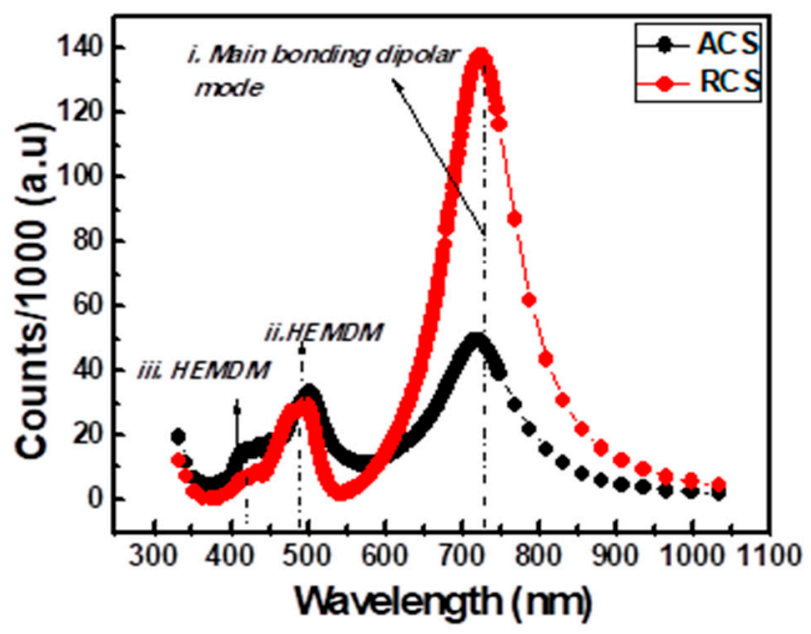

Figure 3. Scattering cross-section of the silver-coated silica nanoparticles as a function of wavelength at $g=0.4 \mathrm{~nm}$.

Figure 3 shows that the scattering cross section for the major dipole resonance mode is high compared to the absorption cross section, while for the other two HEMDM peaks, both types of cross sections are comparable The position of the major dipolar mode is $740 \mathrm{~nm} \sim 3 \lambda_{o} / 2$, while the other two HEMDM peaks indicated by squares and triangles are located at $502 \mathrm{~nm} \sim \lambda_{o}$ and $438 \mathrm{~nm} \sim 6 \lambda_{o} / 7$, where $\lambda_{o}=504 \mathrm{~nm}$ is the reference wavelength and corresponds to the resonance wavelength of a single isolated SCS nanoparticle. These peak positions verify that all three peaks arise due to the co-existence of different dipoles. These dipoles are completely independent from each other, but their generation depends only on the inter-particle distance $g$. Also the small $g$ value causes the surface charges to reorient due to unbalanced Coulombic forces, resulting in a non-uniform charge distribution across the particles surfaces. This charge distribution gradient leads to the generation of two additional dipolar modes with high energy and resonance frequency.

The expected electric field distribution and its directivity at all three resonance wavelengths are shown in Figure 4a-f. Significant enhancement was predicted in the local field inside or in close proximity to the dimer. 

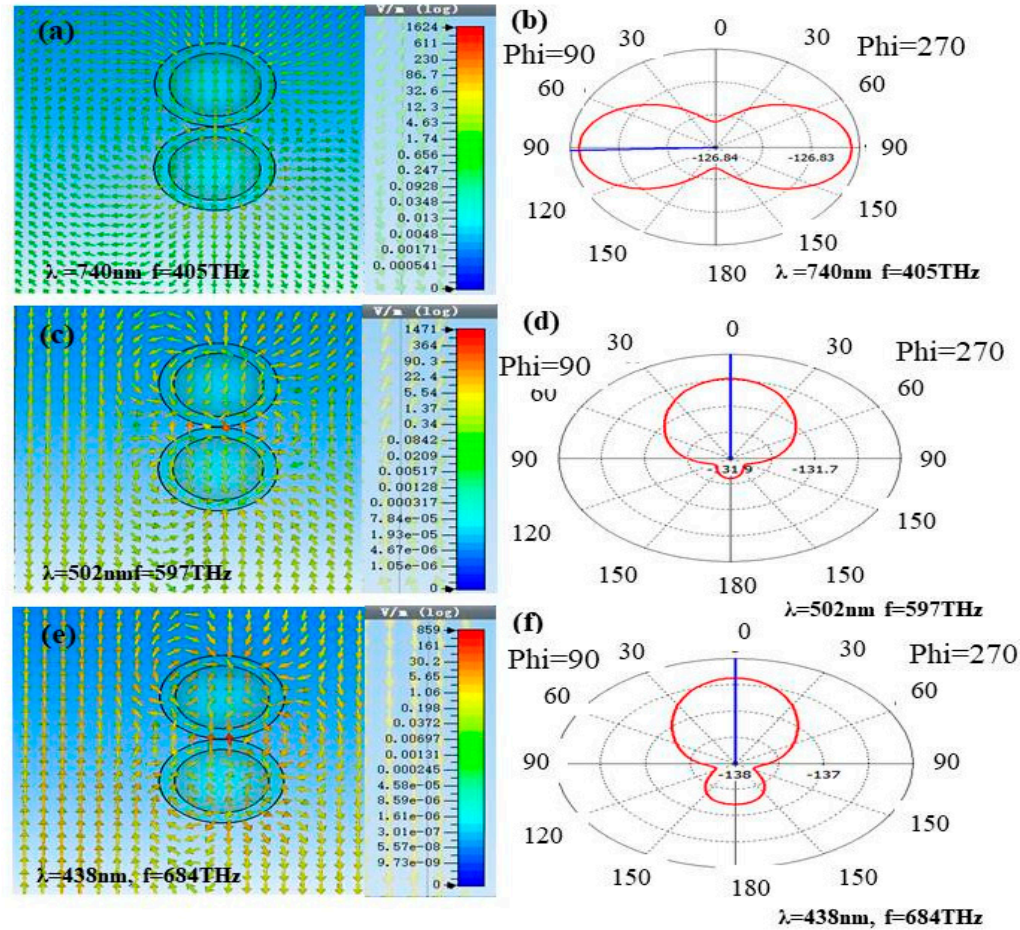

Figure 4. The left vertical panel (a,c,e) shows the current density and strength of various dipolar modes at frequency $f=405 \mathrm{THz}, 597 \mathrm{THz}$ and $684 \mathrm{THz}$ respectively, while the right vertical panel $(\mathbf{b}, \mathbf{d}, \mathbf{f})$ is the directivity of the corresponding dipolar modes.

In Figure $4 \mathrm{a}, \mathrm{c}, \mathrm{e}$, the field vectors pronounce the density and direction of the current, which correspond to the major bonding dipole and HEMDM, respectively, in the SCS dimer at their resonance frequency/wavelength. There is a uniform electric field distribution inside and nearby the SCS dimer during the major dipolar mode where resonance wavelength $\lambda=740 \mathrm{~nm}$. However, the local field in the vicinity of the dimer is stronger than the field of the incident electromagnetic planewave. In the case of HEMDM $(\lambda=502 \mathrm{~nm}, 438 \mathrm{~nm})$, the local field enhancement becomes more significant with a slight phase change. This phase change is more apparent in the directivity plot of the nanoantenna. Interesting directivity of the electric field was observed for all three resonance peaks, as shown in Figure $4 \mathrm{~b}, \mathrm{~d}$,f. It should be noted that the planewave is directed along the $Z$-axis. The inside and outside fields during the major dipolar mode is uniform and is propagating along the positive and negative $X$-axes equally. The scattered electric field during the HEMDM exhibits different behavior. In the HEMDM, a unidirectional scattered field along the $Y$-axis was predicted. There is a small back lobe of the scattered electric field for the HEMDM resonance at $\lambda=438 \mathrm{~nm}$ (Figure $4 \mathrm{f}$ ). This means that the major dipolar and HEMDM corresponds to longitudinal and transverse dipolar modes, respectively. In recent literature, these modes have been predicted to have a gap narrower than $1 \mathrm{~nm}$ and have been attributed to morphology and quantum charge transfer (QCT) [20]. However, our work suggests the existence of such longitudinal and transverse dipolar modes for spherical shell surfaces with a gap $\leq 2 \mathrm{~nm}$. Hence, it is suggested that QCT may affect the strength or intensity of these HEMDMs. Moreover, in our case, such high energy dipolar modes occur at $g$ values of $12 \mathrm{~nm}$, which is high compared to the previously reported value $(g=1-2 \mathrm{~nm})$. The generation of these modes is explainable using a classical Drude model modified by including the size of effects of plasmonic nanostructures.

The far-field characteristics of all three dipolar modes have been depicted independently in Figure $5 \mathrm{a}-\mathrm{c}$. Figure 5 demonstrates that the major bonding dipolar mode is highly radiative compared to the other two high frequency modes of the SCS dimer. Hence, it is suggested that most far-field characteristics are due to the major dipolar mode. While the other two HEMDM resonances cause the 
local field properties inside or in close proximity to the dimer to become stronger, especially in the nano-gap region. From Figure 5a-c, we can conclude that the major dipolar mode and the HEMDM are suitable for utilizing the far-field and near-field properties of SCS dimer antennas. The next section will provide more details about the various parameters or phenomena regarding local field enhancement.
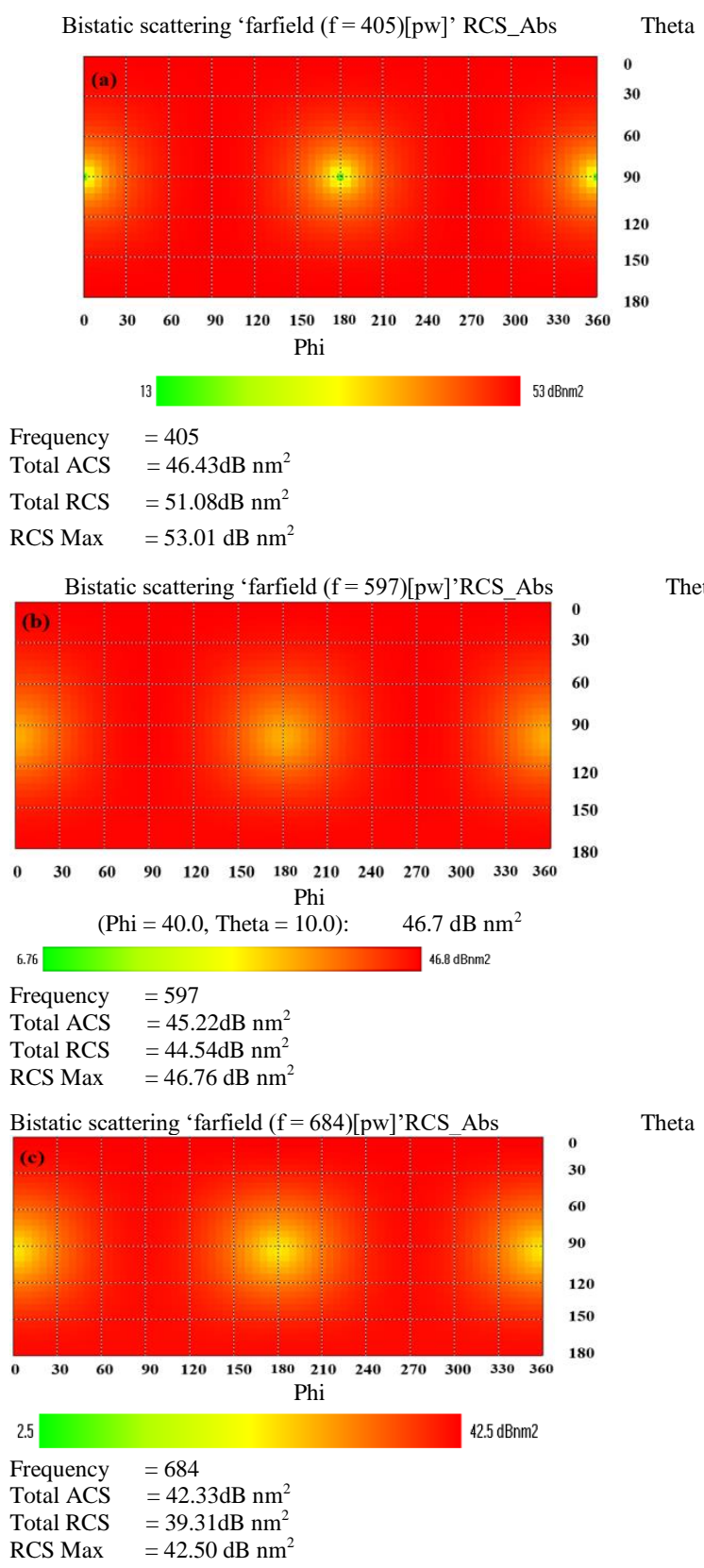

Figure 5. Far-field properties of the SCS dimer. (a) shows the Major bonding dipole at $f=405 \mathrm{THz}$ while $(\mathbf{b}, \mathbf{c})$ are HEMDM at $f=597 \mathrm{THz}$ and $684 \mathrm{THz}$ respectively.

\section{Near Field Coupling and Ruler Equation}

The variation in near-field coupling could be well labeled by a simple dipolar model. According to such a model, the near-field strength strongly depends on the polarizability of the dimer and the decay length constant. Both polarizability and decay constant has an inverse relation with particle volume $D^{3}$ and inter-particle distance $\left(1 / \mathrm{g}^{3}\right)$, respectively [23]. This means that near-field coupling is highly 
sensitive to inter-particle distance normalized by the size of the particle $(r / D)^{-3}$. Hence, the first-order exponential decay Ruler equation has been used to fit the calculated near-field coupling data $[17,24]$ :

$$
\frac{\Delta \lambda}{\lambda_{o}}=A e^{-\frac{g}{\nu}}
$$

The fit parameters $\tau$ and free exponential factor $A$ represent the decay length and near-field coupled strength, respectively. Equation (4) correlates with the fractional plasmonic shift and normalized inter-particle distance $(g / D)$.

The peak position or the fractional plasmonic shift $\left(\Delta \lambda / \lambda_{o}\right)$ of the major plasmonic mode was monitored as a function of inter-particle distance $g$ normalized by particle size $D$, as depicted in Figure 6a; $\Delta \lambda$ and $\lambda_{o}=504 \mathrm{~nm}$ represent the peak shift and reference resonance wavelength of a single SCS nanoparticle, respectively. In this figure, only the shift of the major boning dipole peaks is addressed because the other HEMDM peaks are absent at larger $g$ values. It is clear from Figure 6a that exponential decay Equation (4), with decay constant $\tau=0.2864 \pm 0.091$ and amplitude $A=0.075 \pm 0.05$, describes the calculated data completely with a few exceptions. It should be noted that expected exponential decay does not agree with calculated data when $g / D \leq 0.05 \mathrm{~nm}(g \leq 2 \mathrm{~nm})$. This deviation is due to the breeding of two other HEMDM peaks when nanoparticles are very close to each other $[5,16]$. These modes have already been explained in the aforementioned sections of the article. However, this section includes the deviation of near-field coupling from the exponential Ruler equation due to an abrupt decrease in the fractional plasmonic shift $\left(\Delta \lambda / \lambda_{0}\right)$ and in the intensity of the major dipolar mode. As described earlier, two additional states are generated between the particles of the SCS dimer antenna when $g \leq 2 \mathrm{~nm}$. To understand this, Figure 2 and Table 1 should be consulted. It is clear that two peaks, ii and iii, corresponding to the HEMDM, appear when $g \leq 12 \mathrm{~nm}$ and $g \leq 2$ $\mathrm{nm}$, respectively. In this case, the energy of the incident planewave is divided over all three dipolar modes. This causes the resultant energy of the major bonding dipolar mode to decrease, and as a result the fractional plasmonic phase shift $\left(\Delta \lambda / \lambda_{o}\right)$ becomes negative when the gap $g$ is decreases beyond $2 \mathrm{~nm}$. Obviously, this will affect far-field strength (major dipolar mode), as shown in Figure 6b. Figure $6 \mathrm{~b}$ shows the maximum scattering cross section of the SCS dimer in $\mathrm{dB}$ as a function of the linear inter-particle gap $g$. The maximum scattering cross section (Peak maxima) increases when the $g$ value decreases. However, a sudden fall in the scattering cross section when $g \leq 2 \mathrm{~nm}$ can be seen. The QCT is not included here, because we believe that the QCT may only affect the strength of dipolar modes present in the SCS dimer antenna, but not their origin of generation.

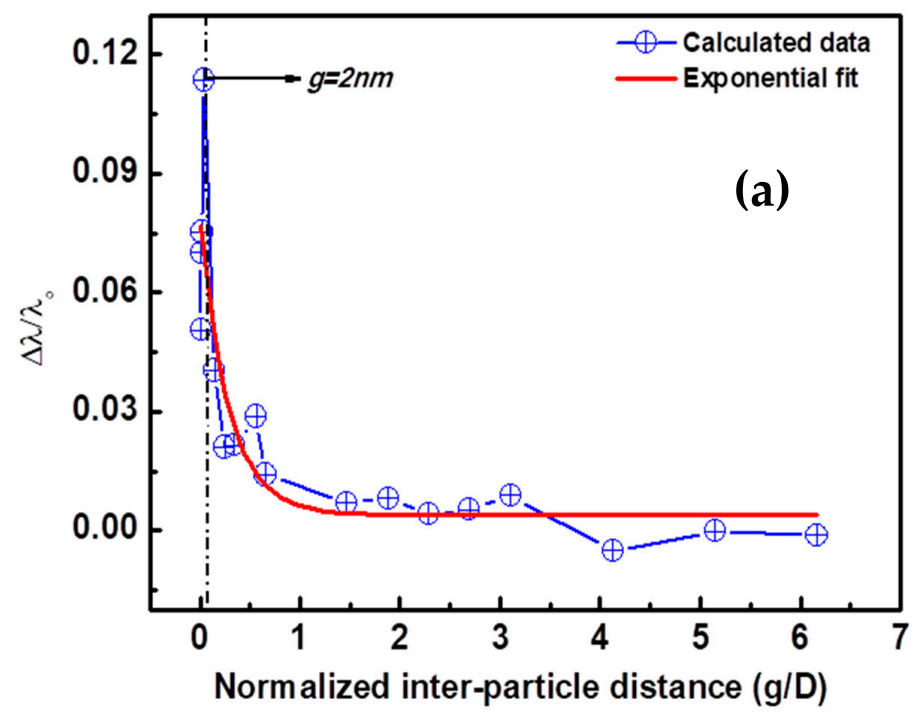

Figure 6. Cont. 


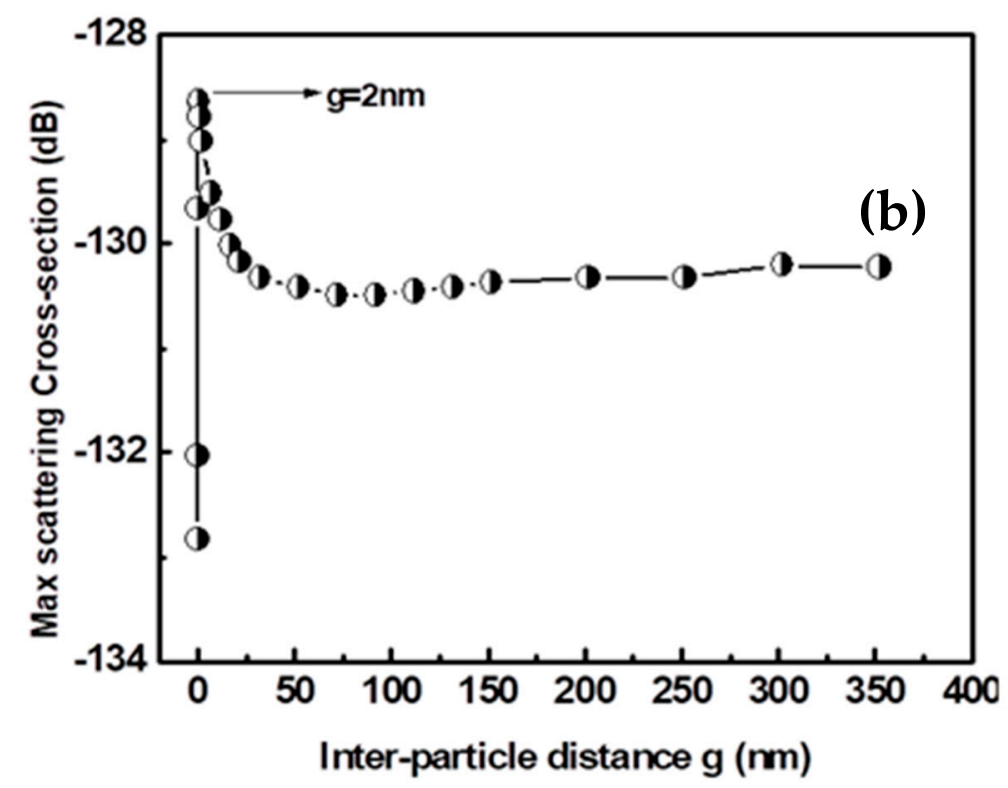

Figure 6. (a) Fractional phase shift vs. normalized inter-particle gap. The green line shows the calculated data when a red one presents the exponential decay Ruler equation fit; (b) Peak maxima as a function of the linear inter-particle gap.

\section{Conclusions}

Numerical analysis via FEM techniques was performed to explain the field coupling of two interacting plasmonic nanoparticles. A major bonding dipolar mode is observed at all given inter-particle gaps within the SCS dimer. However, second and third high energy dipolar resonance peaks were observed at $g=12 \mathrm{~nm}$ and $g=2 \mathrm{~nm}$, respectively. These two additional dipolar modes have a sufficiently high energy or resonance frequency compared to the major bonding dipole. It was found that all dipolar modes have a classical origin and can be explained using a modified Dude model. The modified Drude model incorporates size effects in term of collision frequency. The major dipolar mode contributes greatly to far-field properties, and the HEMDM causes the near-field properties of the dimer antenna to become stronger. It was found that the relation between the fractional phase changes satisfies the exponential decay Ruler equation when $g>2 \mathrm{~nm}$. A breakdown of the Ruler equation along with an abrupt decrease in phase shift and intensity of the major bonding dipolar modes can thus be predicted when the inter-particle gap falls below $2 \mathrm{~nm}$. We suggest that the generation of two modes below a critical value of $g$ has a classical and/or semi-classical origin.

Acknowledgments: This work was supported by the National Natural Science Foundation of China under Grant No. 61571289.

Author Contributions: Atta Ur Rahman wrote and revised this paper. Junping Geng, Xianling Liang and Ronghong Jin provided valuable instructions and assistance to conduct this research work and write this paper. Sami Ur Rehman and Khizar Hayat contributed in calculation of size dependent dielectric constant.

Conflicts of Interest: The authors declare no conflicts of interest

\section{References}

1. Huang, Y.; Zhou, Q.; Hou, M.; Ma, L.; Zhang, Z. Nanogap effects on near- and far-field plasmonic behaviors of metallic nanoparticle dimers. Phys. Chem. Chem. Phys. 2015, 17, 29293-29298. [CrossRef] [PubMed]

2. Gramotnev, D.K.; Bozhevolnyi, S.I. Nanofocusing of electromagnetic radiation. Nat. Photonics 2014, 8, 13-22. [CrossRef]

3. Gramotnev, D.K.; Bozhevolnyi, S.I. Plasmonics beyond the diffraction limit. Nat. Photonics 2010, 4, 83-91. [CrossRef] 
4. Ratner, M.A. Electron Transport in Molecular Wire Junctions. Science 2003, 300, 1384-1389.

5. Søren, R.; Sergey, I.B.; Martijn, W.; Mortensen, N.A. Nonlocal optical response in metallic nanostructures. J. Phys. Condens. Matter 2015, 27, 183204.

6. Stockman, M.I. Nanofocusing of Optical Energy in Tapered Plasmonic Waveguides. Phys. Rev. Lett. 2004, 93, 137404. [CrossRef] [PubMed]

7. Geng, J.; Ziolkowski, R.W.; Jin, R.; Liang, X. Active cylindrical coated nano-particle antennas: Polarization-dependent scattering properties. J. Electromagn. Waves Appl. 2013, 27, 1392-1406. [CrossRef]

8. Jain, P.K.; Huang, W.; El-Sayed, M.A. On the Universal Scaling Behavior of the Distance Decay of Plasmon Coupling in Metal Nanoparticle Pairs: A Plasmon Ruler Equation. Nano Lett. 2007, 7, 2080-2088. [CrossRef]

9. Pileni, M.P. Nanocrystal Self-Assemblies: Fabrication and Collective Properties. J. Phys. Chem. B 2001, 105, 3358-3371. [CrossRef]

10. Wang, Y.; Xie, X.; Wang, X.; Ku, G.; Gill, K.L.; O’Neal, D.P.; Wang, L.V. Photoacoustic Tomography of a Nanoshell Contrast Agent in the in Vivo Rat Brain. Nano Lett. 2004, 4, 1689-1692. [CrossRef]

11. Dodson, S.; Haggui, M.; Bachelot, R.; Plain, J.; Li, S.; Xiong, Q. Optimizing Electromagnetic Hotspots in Plasmonic Bowtie Nanoantennae. J. Phys. Chem. Lett. 2013, 4, 496-501. [CrossRef] [PubMed]

12. Durr, N.J.; Larson, T.; Smith, D.K.; Korgel, B.A.; Sokolov, K.; Ben-Yakar, A. Two-Photon Luminescence Imaging of Cancer Cells Using Molecularly Targeted Gold Nanorods. Nano Lett. 2007, 7, 941-945. [CrossRef] [PubMed]

13. Hooshmand, N.; Bordley, J.A.; El-Sayed, M.A. The Sensitivity of the Distance Dependent Plasmonic Coupling between Two Nanocubes to their Orientation: Edge-to-Edge versus Face-to-Face. J. Phys. Chem. C 2016, 120, 4564-4570. [CrossRef]

14. Weeraddana, D.; Premaratne, M.; Andrews, D.L. Quantum electrodynamics of resonance energy transfer in nanowire systems. Phys. Rev. B 2016, 93, 075151. [CrossRef]

15. Hanson, G.W. Drift-Diffusion: A Model for Teaching Spatial-Dispersion Concepts and the Importance of Screening in Nanoscale Structures. IEEE Antennas Propag. Mag. 2010, 52, 198-207. [CrossRef]

16. Mortensen, N.A.; Raza, S.; Wubs, M.; Søndergaard, T.; Bozhevolnyi, S.I. A generalized non-local optical response theory for plasmonic nanostructures. Nat. Commun. 2014, 5, 3809. [CrossRef] [PubMed]

17. Su, K.H.; Wei, Q.H.; Zhang, X.; Mock, J.J.; Smith, D.R.; Schultz, S. Interparticle Coupling Effects on Plasmon Resonances of Nanogold Particles. Nano Lett. 2003, 3, 1087-1090. [CrossRef]

18. Zhu, W.; Esteban, R.; Borisov, A.G.; Baumberg, J.J.; Nordlander, P.; Lezec, H.J.; Crozier, K.B. Quantum mechanical effects in plasmonic structures with subnanometre gaps. Nat. Commun. 2016, 7, 11495. [CrossRef] [PubMed]

19. Hooshmand, N.; Bordley, J.A.; El-Sayed, M.A. Are Hot Spots between Two Plasmonic Nanocubes of Silver or Gold Formed between Adjacent Corners or Adjacent Facets? A DDA Examination. J. Phys. Chem. Lett. 2014, 5, 2229-2234. [CrossRef] [PubMed]

20. Esteban, R.; Aguirregabiria, G.; Borisov, A.G.; Wang, Y.M.; Nordlander, P.; Bryant, G.W.; Aizpurua, J. The Morphology of Narrow Gaps Modifies the Plasmonic Response. ACS Photonics 2015, 2, $295-305$. [CrossRef]

21. Geng, J.; Ziolkowski, R.W.; Jin, R.; Liang, X. Numerical Study of the Near-Field and Far-Field Properties of Active Open Cylindrical Coated Nanoparticle Antennas. IEEE Photonics J. 2011, 3, 1093-1110. [CrossRef]

22. Qiu, W.; DeLacy, B.G.; Johnson, S.G.; Joannopoulos, J.D.; Soljačić, M. Optimization of broadband optical response of multilayer nanospheres. Opt. Express 2012, 20, 18494-18504. [CrossRef] [PubMed]

23. Bordley, J.A.; Hooshmand, N.; El-Sayed, M.A. The Coupling between Gold or Silver Nanocubes in Their Homo-Dimers: A New Coupling Mechanism at Short Separation Distances. Nano Lett. 2015, 15, 3391-3397. [CrossRef] [PubMed]

24. Mock, J.J.; Hill, R.T.; Degiron, A.; Zauscher, S.; Chilkoti, A.; Smith, D.R. Distance-Dependent Plasmon Resonant Coupling between a Gold Nanoparticle and Gold Film. Nano Lett. 2008, 8, 2245-2252. [CrossRef] [PubMed]

(C) 2017 by the authors. Licensee MDPI, Basel, Switzerland. This article is an open access article distributed under the terms and conditions of the Creative Commons Attribution (CC BY) license (http:/ / creativecommons.org/licenses/by/4.0/). 